\title{
Cadena de suministros: Una alternativa de planificación de productos en el sector industrial de Colombia
}

\section{Supply chain: An alternative of product planning in the industrial sector of Colombia}

\author{
Elkin Baleta-Araujo \\ elkinbaleta@unicesar.edu.co \\ Universidad Popular del Cesar, Valledupar Cesar \\ Colombia \\ https://orcid.org/0000-0002-2942-7281 \\ Aneleth Jasbleydi Olmedo-Plata \\ aneletholmedo@unicesar.edu.co \\ Universidad Popular del Cesar, Valledupar Cesar \\ Colombia \\ https://orcid.org/0000-0001-9090-0560
}

Recibido: 08 de abril de 2020

Revisado: 10 de mayo de 2020

Aprobado: 2 de junio de 2020

Publicado: 09 de junio de 2020

\begin{abstract}
RESUMEN
La investigación que se desarrolló tuvo como objeto analizar la cadena de suministros como una alternativa de planificación de productos en el sector industrial de Colombia. La misma es de carácter descriptivo, ya que percibe la descripción, registro, análisis e interpretación de la naturaleza actual, composición o procesos de los fenómenos, además del investigador quien se involucra de manera objetiva y fiable en el proceso investigativo; Asimismo, se realizó una revisión documental sobre las cadenas de suministro para presentar la evolución y tendencias futuras de las operaciones, sustentándose en teorías referenciales de autores como Carrasco, (2010); Young y Esqueda, (2015), entre otros. Como conclusión, se afirmar que las cadenas de abastecimiento ofrecen ventajas competitivas a las empresas quienes se han visto en la necesidad de desarrollarlas y mantenerlas actualizadas.
\end{abstract}

Descriptores: Cadena de Suministros; productos; sector industrial; ventajas competitivas. 


\author{
CIENCIAMATRIA \\ Revista Interdisciplinaria de Humanidades, Educación, Ciencia y Tecnología \\ Año VI. Vol. VI. N¹1. Julio - Diciembre 2020 \\ Hecho el depósito de ley: pp201602FA4721 \\ ISSN-L: 2542-3029; ISSN: 2610-802X \\ Universidad Nacional Experimental Francisco de Miranda (UNEFM). Santa Ana de Coro. Venezuela
}

Elkin Baleta-Araujo; Aneleth Jasbleydi Olmedo-Plata

\begin{abstract}
The research that was carried out was aimed at analyzing the supply chain as an alternative for product planning in the Colombian industrial sector. It is descriptive in nature, since it perceives the description, registration, analysis and interpretation of the current nature, composition or processes of the phenomena, in addition to the researcher who engages objectively and reliably in the investigative process; Likewise, a documentary review of supply chains was carried out to present the evolution and future trends of operations, based on referential theories by authors such as Carrasco, (2010); Young and Esqueda, (2015), among others. In conclusion, it is stated that supply chains offer competitive advantages to companies who have seen the need to develop and keep them up-to-date.
\end{abstract}

Descriptors: Supply chain; products, industrial sector; competitive advantages.

\title{
INTRODUCCIÓN
}

La logística de operaciones, un concepto ampliamente utilizado, ha ido cambiando en el ámbito empresarial evolucionando hacia lo que hoy se conoce como cadena de suministros, la cual en función de lo planteado por los autores Carrasco, (2010); Young y Esqueda, (2015) es la unión de varios eslabones de carácter logístico que integran la empresa, estas áreas logísticas que se fusionan bajo una nueva gerencia son: pronóstico de la demanda, planeación de ventas, operaciones, y compromisos con clientes; esta nueva dirección busca aumentar la competitividad mediante un aumento de la productividad, generado por la optimización de sus procesos logísticos aunado a un aumento del nivel de servicio al cliente, minimizando los costos operativos, todos estos procesos integrados a través de un flujo de información.

Por lo anterior, según Fernández (2008) es importante conocer sobre un concepto y toda una metodología de trabajo que muchas empresas han olvidado, como lo es la cadena de suministros, práctica que permite a las organizaciones mejorar los procesos internos para así mantener su competitividad, satisfacer totalmente el mercado y gerenciar adecuadamente procesos propios y externos que afectan la organización.

Con todo lo expuesto, es posible lograr que las compañías no fracasen y se mantengan exitosamente. Pero en la práctica de la cadena de suministros, no sólo 


\section{CIENCIAMATRIA}

Revista Interdisciplinaria de Humanidades, Educación, Ciencia y Tecnología

Año VI. Vol. VI. N¹1. Julio - Diciembre 2020

Hecho el depósito de ley: pp201602FA4721

ISSN-L: 2542-3029; ISSN: 2610-802X

Universidad Nacional Experimental Francisco de Miranda (UNEFM). Santa Ana de Coro. Venezuela

\section{Elkin Baleta-Araujo; Aneleth Jasbleydi Olmedo-Plata}

se mejoran los procesos internos, sino también los externos. Debido al entorno agresivo que actualmente se presenta para una organización, es necesario que se tenga en cuenta también, dentro del desarrollo de sus procesos, la relación que se tiene con los proveedores y los clientes de la empresa, es aquí donde la cadena de suministros da un valor agregado extendiendo las fronteras de las compañías. Por tanto, señalan Martínez y Elkadi (2018), la capacidad de la función logística de coordinarse e integrarse con los demás áreas de la organización, es esencial para responder con las necesidades en la cadena de suministro.

\section{Referentes Teóricos}

\section{De logística de operaciones a cadena de suministro}

La cadena de suministro es la evolución de las operaciones logísticas a nivel empresarial, entre las definiciones de Logística de Operaciones, están; Para el Consejo de Profesionales en Administración de la Cadena de Suministros (CSCMP 2010, por sus siglas en inglés), se define logística como el proceso de planificación, ejecución y control de los procedimientos necesarios para el manejo eficiente y eficaz de las mercancías, tanto en el transporte como en el almacén, incluyendo el servicio de información desde el origen y hasta los clientes.

En este sentido, Quiroga (2009), define logística como el proceso de planificar, implementar y controlar el flujo y almacenamiento de materiales, productos en proceso o terminados, así como el manejo de la información desde el lugar de origen hasta el lugar de consumo, con el propósito de satisfacer los requerimientos de los clientes.

De acuerdo con esto, se habla de operaciones logística al proceso de gestión (entendido como el ciclo de planear, hacer, verificar y actuar) de los flujos de productos, servicios e información relacionados con el proceso productivo. Nickl (2005), distingue una logística tradicional la cual comprende el movimiento físico de materiales y donde las áreas como compras o producción, comercial o ventas, son las que definen su ámbito de actuación; ésta tiene como objetivo administrar los inventarios que se generan por las inflexibilidades relacionadas con las compras o a 


\section{CIENCIAMATRIA}

Revista Interdisciplinaria de Humanidades, Educación, Ciencia y Tecnología

Año VI. Vol. VI. N¹1. Julio - Diciembre 2020

Hecho el depósito de ley: pp201602FA4721

ISSN-L: 2542-3029; ISSN: 2610-802X

Universidad Nacional Experimental Francisco de Miranda (UNEFM). Santa Ana de Coro. Venezuela

\section{Elkin Baleta-Araujo; Aneleth Jasbleydi Olmedo-Plata}

la producción. En relación a las áreas comerciales o de venta su misión es mover los productos, dentro de los parámetros establecidos, hacia los clientes.

Con base en las investigaciones de Young y Esqueda (2015), la teoría de cadena de suministro surge en la década de los sesenta, cuando las empresas deciden unir actividades relacionadas creando las gerencias de distribución física, la cual resultó de la unión de las gerencias de inventario, almacenamiento, despacho de producto terminado y servicio al cliente. También emerge la gerencia de materiales, la cual estaría compuesta de la gestión de compras, recepción e inventarios de materias primas, así como planificación y control de la producción.

Luego en la década de los setenta, las gerencias de distribución física y materiales se combinan originando la gerencia de logística; en dicho momento se evidencia la necesidad de manejar un flujo de información paralelo al flujo de productos manejados, por lo que nace una gerencia integral de logística. Posteriormente, en la década de los noventa, las empresas reconocen que los flujos financieros entre compradores y vendedores operan de forma más eficiente cuando el flujo físico y de información son manejados eficientemente, siendo este el primer paso para la gerencia de la cadena de suministro.

Considerando lo planteado, Gutiérrez, Fuquen y González, (2010) comentan que la cadena de suministro está formada por la integración de todas las áreas funcionales necesarias para satisfacer las necesidades de los clientes, abarcando los flujos de materiales desde el proveedor hasta la entrega al cliente y los servicios post venta. Esta combinación de funciones se visualiza a través de tres flujos: materiales, monetarios e información, los cuales determinan la configuración de la cadena de suministro.

Los flujos de materiales se refieren al movimiento de los materiales y abarca los traslados, espacios de almacenamiento, e inventario; el de información da relación del estado físico de los tangibles a la organización, mientras que los monetarios, se originan por la relación de los anteriores, las fluctuaciones económicas, políticas gubernamentales, acuerdos operativos, entre otros. 


\section{CIENCIAMATRIA}

Revista Interdisciplinaria de Humanidades, Educación, Ciencia y Tecnología

Año VI. Vol. VI. N¹1. Julio - Diciembre 2020

Hecho el depósito de ley: pp201602FA4721

ISSN-L: 2542-3029; ISSN: 2610-802X

Universidad Nacional Experimental Francisco de Miranda (UNEFM). Santa Ana de Coro. Venezuela

\section{Elkin Baleta-Araujo; Aneleth Jasbleydi Olmedo-Plata}

\section{Integración de la Cadena de Suministro}

Hoy en día, la forma de hacer frente a los negocios requiere de un enfoque que va más allá de las fronteras de la empresa, para trabajar en equipo con clientes y proveedores para mejorar la relación costo/servicio como una cadena, no como una empresa aislada, trasladando así múltiples beneficios a los consumidores finales quienes son los "dueños del poder". Con lo que se determina la importancia de la integración entre empresas como el punto estratégico y clave más importante en la administración de las cadenas de suministro. Para lograrla efectivamente, según Ballesteros y Ballesteros (2014) se deben tener en cuenta cuatro tipos de integración que usados a la vez determinan el nivel y la capacidad de una empresa para optimizar sus cadenas de suministro. Estos tipos de integración son:

- Integración funcional de las actividades de compras, manufactura, transporte, manejo de inventarios y almacenamiento.

Integración espacial a través de la dispersión geográfica de proveedores, fábricas, centros de distribución y clientes.

- Integración inter-temporal de las actividades de planeación estratégica, táctica y operativa de cada empresa y en conjunto de cada cadena.

- Integración empresarial que incluye los planes de la cadena de suministros, los planes de mercadeo y ventas y los planes financieros.

Con los proveedores existe una coordinación metódica de los programas de producción con los programas de suministros, la cual se planifica conjuntamente con los proveedores para introducir mejoras en su proceso, a fin de aumentar o agregar valor a los suministros. Por otra parte, existe un intercambio de información sistemática con los proveedores y se cuenta con una adecuada integración de la tecnología de información. Así mismo, se observa unificación e integración de la técnica y medios de identificación de las cargas con los proveedores y los clientes. Con los clientes existe una coordinación sistemática para ajustar los programas de distribución y mejorar el servicio al cliente. Se controla y monitorea a través de un sistema formal el comportamiento del servicio a los clientes y se establecen programas de mejoramiento. Se brinda una atención personalizada a los clientes. El 


\section{CIENCIAMATRIA}

Revista Interdisciplinaria de Humanidades, Educación, Ciencia y Tecnología

Año VI. Vol. VI. N¹1. Julio - Diciembre 2020

Hecho el depósito de ley: pp201602FA4721

ISSN-L: 2542-3029; ISSN: 2610-802X

Universidad Nacional Experimental Francisco de Miranda (UNEFM). Santa Ana de Coro. Venezuela

\section{Elkin Baleta-Araujo; Aneleth Jasbleydi Olmedo-Plata}

cliente mantiene una relación permanente con la empresa y tiene acceso en cualquier momento a la información sobre el estado de su pedido y sobre el proceso productivo del mismo.

Para garantizar lo anterior, se establecen políticas, procedimientos y estándares en conjunto con los proveedores y clientes para lograr racionalidad y optimización a nivel de toda la cadena de suministro, facilitando la conectividad entre los sistemas informativos de la empresa con los de los proveedores y clientes, apoyándose en una elevada estandarización de los formatos de información y los medios utilizados, con el criterio de mejorar el servicio conjuntamente con una mayor racionalización de los procesos logísticos.

De allí entonces que según lo expresado por Vivar, Erazo y Narváez (2020) es importante que las empresas mantengan una ventaja competitiva obtenida mediante la correcta ejecución de la cadena de valor, la cual determina varios factores como el costo, la calidad, la respuesta a tiempo, y flexibilidad, esto conlleva a la rentabilidad de la organización.

\section{Organización de la producción y el surgimiento de la cadena logística de suministro}

En el mundo del comercio internacional y empresarial existente se ha originado un giro de enorme trascendencia en la gestión de la producción y en el intercambio de bienes. Es por ello que, Jiménez y Hernández (2012) expresan que tal situación originó la dispersión de la producción y la ampliación geográfica de los mercados, lo cual ha conformado grandes sistemas de producción y complejos esquemas operativos para atender los flujos mundiales de demanda de mercancías en un entorno económico excesivamente dinámico.

Dicho entorno económico no tardó en dejar sentir sus efectos directos sobre el territorio. La anticuada organización productiva y distributiva se está modificando para buscar un funcionamiento más adecuado a las nuevas exigencias. En términos generales, se puede decir que en los últimos tres decenios, el entorno (ciudades y 


\section{CIENCIAMATRIA}

Revista Interdisciplinaria de Humanidades, Educación, Ciencia y Tecnología

Año VI. Vol. VI. N¹1. Julio - Diciembre 2020

Hecho el depósito de ley: pp201602FA4721

ISSN-L: 2542-3029; ISSN: 2610-802X

Universidad Nacional Experimental Francisco de Miranda (UNEFM). Santa Ana de Coro. Venezuela

\section{Elkin Baleta-Araujo; Aneleth Jasbleydi Olmedo-Plata}

territorios) experimentó alteraciones de un alcance al que anteriormente no se había llegado en el curso del siglo XX.

En casos especiales, ante este escenario, se puede afirmar que modelos como el "centro - periferia" comienzan a sucumbir. Dicha transformación significa simplemente la desintegración del sistema de organización de la producción tradicional, por un sistema nuevo que involucra con mayor profundidad la gestión logística.

Como sede de la producción, el territorio se modifica según la nueva articulación logística de los eslabones de la producción y de los flujos físicos de las mercancías. Dicha alteración del territorio está afectada también por el establecimiento de redes logísticas de suministro y distribución de la producción, que sustituyen los esquemas existentes, articulando el sistema productivo con el mercado por medio de la función logística. Desde luego, es innegable que dicho proceso esté ligado a la recíproca articulación de los factores de la producción, el capital y el trabajo.

Haciendo un paréntesis, los modelos clásicos de emplazamiento industrial de Thunen (1826), Hoover (1888), Weber (1909), Lorsh (1900), Christaller (1933), determinaron que la localización industrial estaba definida por la ubicación de las materias primas y los recursos energéticos. Por una parte, los recursos extractivos (carbón y minerales de hierro, en particular ), y por el otro los cursos de agua, que se podían emplear para obtener fuerza motriz y para eliminar o dar salida a los subproductos de las elaboraciones industriales, dando paso a una configuración centralizada de la producción, denominada por Marshall (2008) como aglomeración o distritos industriales.

Dichos modelos surgieron con enfoques puramente económicos basados en: costos mínimos (transporte, producción y costos totales); en ingresos brutos (orientados al mercado más favorable y mayores ingresos brutos); y en utilidades máximas, modelos clásicos con notables relaciones del uso del suelo.

Siguiendo con el citado autor, señala que la existencia de plantas industriales en ciertas zonas provocó a su vez la atracción de nuevas inversiones que estimularon el crecimiento económico regional. De esta manera, Krugman, (2012) comenta que el 


\section{CIENCIAMATRIA}

Revista Interdisciplinaria de Humanidades, Educación, Ciencia y Tecnología

Año VI. Vol. VI. N¹1. Julio - Diciembre 2020

Hecho el depósito de ley: pp201602FA4721

ISSN-L: 2542-3029; ISSN: 2610-802X

Universidad Nacional Experimental Francisco de Miranda (UNEFM). Santa Ana de Coro. Venezuela

\section{Elkin Baleta-Araujo; Aneleth Jasbleydi Olmedo-Plata}

inventario de las materias primas y un mercado consumidor fueron los ejes fundamentales del emplazamiento empresarial y la base de la teoría clásica de la localización, dando pie al mismo tiempo a las teorías de la aglomeración y a una nueva organización de la interrelación de las unidades de producción. Es decir, que la concentración geográfica surge, básicamente, de la interrelación de los rendimientos crecientes, los costos de transporte y la demanda

La idea de que las fábricas se concentraran en sitios cercanos a los lugares de procedencia de la materia prima, de los mercados potenciales (consumidores) y a las economías de aglomeración y de escala, desde luego no era mala, sin embargo, la revisión de los procesos de aprovisionamiento, producción y distribución fueron poco desarrollados para maximizar el beneficio. Una vez definido el emplazamiento "ideal", uno de los principales enfoques complementarios de dichas teorías era que se centraban más en la producción que en el consumo, y el sector de los servicios se pudo observar que era tratado como una esfera de actividad aislada.

Bajo este estigma, Giménez (2010) mención que la teoría clásica relegaba la gestión logística a un segundo plano en la toma de decisiones para el emplazamiento industrial, pues como se dijo anteriormente, la organización territorial de la producción obedecía exclusivamente a criterios económicos, buscando determinar el lugar ideal para localizar una planta en el cual se pudiesen conseguir los materiales, realizar los procesos y distribuir los productos con el mínimo costo total e inconveniencias, tomando en cuenta un único parámetro conocido, la distancia.

Aceptar del todo los principios de la fragmentación industrial en el espacio regional, bajo el nuevo paradigma de la organización logística industrial requerirá de encontrar respuestas a las siguientes preguntas: ¿cuáles son los factores o criterios más relevantes que hoy en día consideran las empresas para conformar acuerdos comerciales que derivan en la formación de las cadenas logísticas de suministro?, ¿de qué manera han cambiado los criterios de emplazamiento en un entorno de competitividad en la conformación de cadenas de suministro?, ¿el transporte sigue representando un elemento de peso en el emplazamiento industrial y en la conformación de dichas cadena?, ¿están preparados, tanto usuario de transporte 


\section{CIENCIAMATRIA}

Revista Interdisciplinaria de Humanidades, Educación, Ciencia y Tecnología

Año VI. Vol. VI. N¹1. Julio - Diciembre 2020

Hecho el depósito de ley: pp201602FA4721

ISSN-L: 2542-3029; ISSN: 2610-802X

Universidad Nacional Experimental Francisco de Miranda (UNEFM). Santa Ana de Coro. Venezuela

\section{Elkin Baleta-Araujo; Aneleth Jasbleydi Olmedo-Plata}

como proveedores del servicio para adoptar la nueva filosofía de la logística colaborativa?, yendo más lejos, ¿este nuevo enfoque puede ser una mecanismo de desarrollo regional?, ¿de organización de la producción?, ¿puede ayudar a lograr una mejora sustancial en la competitividad?.

Dar respuesta a dichos planteamientos desde luego no es una tarea fácil, sin embargo, sin caer en una situación simplista de un tema con alto contenido de debate, se puede observar que a partir de la dispersión de la producción que se está viviendo, se aprecia el surgimiento de una nueva interrelación entre las unidades productivas, el territorio y el transporte, dando paso a una posible evolución de las teorías de emplazamiento y la teoría logística.

\section{Alcance de la cadena de suministro}

El alcance de la cadena de suministro se puede definir según Jiménez y Hernández (2012) como el rango o nivel más alejado de proveedores y clientes que tienen cierto grado de influencia en los procesos productivos y logísticos de la planta. En principio, el alcance de la cadena de suministro según Arenas (2011) puede abarcar a todas aquellas unidades de negocio que intervienen en el proceso de producción y consumo. Sin embargo, en un esquema de análisis más estricto, es importante considerar otros elementos que podrían ser estratégicos y fundamentales, externos a dicho proceso.

En el caso del transporte, este elemento juega un papel relevante en la cadena de suministro puesto que permite conjugar las actividades logísticas y de él depende mucho el funcionamiento adecuado del sistema. En la práctica, debido a que los proveedores de servicio no agregan valor económico al producto, en el análisis de la cadena de suministro es común encontrar que no se le otorga la debida importancia, e incluso son actividades relegadas a departamentos que no tienen injerencia.

Como ya se mencionó, los niveles (o rangos) básicamente se distinguen con la finalidad de conocer el alcance del análisis de la cadena de suministro, pero ello también ayuda a distinguir la jerarquía de los eslabones en la misma Es decir, se debe tener en mente que la cadena de suministro es un sistema complejo y que está 


\section{CIENCIAMATRIA}

Revista Interdisciplinaria de Humanidades, Educación, Ciencia y Tecnología

Año VI. Vol. VI. N¹1. Julio - Diciembre 2020

Hecho el depósito de ley: pp201602FA4721

ISSN-L: 2542-3029; ISSN: 2610-802X

Universidad Nacional Experimental Francisco de Miranda (UNEFM). Santa Ana de Coro. Venezuela

\section{Elkin Baleta-Araujo; Aneleth Jasbleydi Olmedo-Plata}

integrada por subsistemas más pequeños, esto se debe entender como la introducción de sistemas en otros sistemas.

Específicamente, las empresas persiguen varios fines y desempeñan muchas funciones. La función primaria de la empresa siempre será elaborar su producto y prestar un servicio con ánimo de lucro. Para Porter, (2010) Con los nuevos paradigmas de la competitividad, la integración de la empresa con sus proveedores y clientes ocupa la función secundaria, con la plena intención de lograr mayor seguridad como miembro de la cadena de suministro. En la generalidad de las empresas, una característica central de la competencia es que son mutuamente dependientes. Las empresas perciben los efectos de los movimientos de unas y otras y están propensas a reaccionar ante ello.

En un sistema de cadena de suministro lo anterior podría ser más crítico. Si en algún momento dado, un elemento importante del sistema pretende un cambio que haga peligrar la estabilidad del sistema, el efecto en la cadena se potencia porque la repercusión es directa sobre dicho elemento y en el resto de los eslabones. El desarrollo y aplicación de una mejor tecnología, así como la innovación logística, son algunos de los factores que podrían provocar un mayor impacto en la cadena. Incluso, el acceso a nuevos participantes (o la salida) de la cadena puede influir en el funcionamiento global del sistema.

La falta de análisis de las consecuencias de las decisiones por parte de los eslabones de la cadena en el largo plazo puede provocar un deterioro paulatino y cada vez mayor del sistema. Por ejemplo, la decisión unilateral de una empresa de la cadena por reducir sus inventarios y fabricar sobre pedido, parece ser una acción no muy acertada, pues en una fase alcista de la demanda, la empresa podría correr el riesgo de verse saturada de pedidos a los que sólo podrá responder con grandes retrasos, provocando que algunos de sus clientes recurran a la competencia y que quede fuera de la cadena de suministro.

En relación a lo expuesto, Porter, (2010) expresa que el alcance de la cadena de suministro toma su lugar cuando las decisiones individuales de uno o un subconjunto de eslabones tienen impacto en el resto de los eslabones. De igual modo, el alcance 


\section{CIENCIAMATRIA}

Revista Interdisciplinaria de Humanidades, Educación, Ciencia y Tecnología

Año VI. Vol. VI. N¹1. Julio - Diciembre 2020

Hecho el depósito de ley: pp201602FA4721

ISSN-L: 2542-3029; ISSN: 2610-802X

Universidad Nacional Experimental Francisco de Miranda (UNEFM). Santa Ana de Coro. Venezuela

\section{Elkin Baleta-Araujo; Aneleth Jasbleydi Olmedo-Plata}

se refleja en la capacidad de respuesta de cada empresa la cual determina la capacidad de respuesta de toda la cadena, por tanto, el eslabón más débil condiciona la fortaleza y alcance de la misma. Asimismo, el alcance de una cadena de suministro está limitado por el tipo de producto y casi siempre por el enfoque de la empresa. Desde el punto de vista de la teoría de las restricciones, el alcance de la cadena de suministro puede ser extendido identificando las restricciones del sistema. La aplicación de esta teoría en la optimización del funcionamiento de un sistema de red de empresas, parte de la identificación de los "cuellos de botella" buscando equilibrar el flujo, no la capacidad del sistema de empresas. En términos de Goldratt (1979), en un sistema de red, las empresas intentan individualmente aumentar la capacidad de tal o cual recurso esperando con ello ser más "competitivo" cuando en realidad no es precisamente la mejor alternativa. Desde el punto de vista de la cadena de suministro la mayor eficiencia de la empresa en su conjunto vendrá de una mayor fluidez y sincronización de los flujos.

A manera de conclusión, la existencia de situaciones de alta "complejidad" en un entorno globalizado, plantea problemas a las empresas que las obliga a buscar su integración en cadenas de suministro para tomar mejores decisiones utilizando flujos de información más claros y confiables. Tal integración impone una mayor diferenciación funcional del alcance del sistema; verticalmente: articulado en varios niveles jerárquicos; y horizontalmente: articulándose con distintas empresas funcionales. De allí entonces que se hace importante destacar a Urrego (2020) la investigación colaborará con las organizaciones en dos niveles el primero referido a buscar la eficacia y eficiencia en los procesos, segundo referido a la documentación requerida para cultivar la enseñanza o la formación de profesionales en el área gerencial. 


\section{CIENCIAMATRIA}

Revista Interdisciplinaria de Humanidades, Educación, Ciencia y Tecnología

Año VI. Vol. VI. N¹1. Julio - Diciembre 2020

Hecho el depósito de ley: pp201602FA4721

ISSN-L: 2542-3029; ISSN: 2610-802X

Universidad Nacional Experimental Francisco de Miranda (UNEFM). Santa Ana de Coro. Venezuela

\section{Elkin Baleta-Araujo; Aneleth Jasbleydi Olmedo-Plata}

\section{METODOLOGÍA}

Esta investigación es de tipo descriptiva, según Tamayo (2009), ya que comprende la descripción, registro, análisis e interpretación de la naturaleza actual, composición o procesos de los fenómenos. Planteándose como un diseño documental; se estará revisando la bibliografía y apoyar la formación de un profesional dentro de lo que hoy se conoce como sociedad del conocimiento, donde se le imparte una formación formal pero le abre una nueva visión para impulsar el aprender a lo largo de su gestión.

Tal como lo señalan Eskandari et al. (2007), en su estudio, donde recalcan la necesidad de incorporar en los planes de estudios de Ingeniería Industrial, temas de gestión y liderazgo, los relacionados con ingeniería de calidad y gestión de la cadena de suministro; considerando como importante que los futuros profesionales tengan entre sus atributos la disponibilidad al entrenamiento en los procedimientos relativos a cada organización.

En esta investigación, se realiza una revisión documental sobre las cadenas de suministro. La misma tiene como objetivo presentar la evolución y tendencias futuras de las operaciones, así como una reflexión sobre la Ingeniería Industrial y sus requerimientos futuros en el ámbito de la logística, área donde las empresas están buscando integrarse para aumentar su competitividad.

\section{REFLEXIONES FINALES}

En la actualidad, uno de los objetivos más buscados por todas las empresas es la mayor eficiencia al menor costo, sin dejar por un lado los estándares de calidad y servicio al cliente. Dichos estándares (métricas) deben ser monitoreados y controlados a lo largo de todo el proceso, desde el origen al término de la cadena de suministros. Este control no solo ayuda a reducir costos, sino que a largo plazo se convierte en una ventaja competitiva.

Es por ello, que con base a las ideas expuestas, el análisis e interpretación de los distintos conceptos abordados en este trabajo, surgen algunas conclusiones que se presentan a continuación: 


\section{CIENCIAMATRIA}

Revista Interdisciplinaria de Humanidades, Educación, Ciencia y Tecnología

Año VI. Vol. VI. N¹1. Julio - Diciembre 2020

Hecho el depósito de ley: pp201602FA4721

ISSN-L: 2542-3029; ISSN: 2610-802X

Universidad Nacional Experimental Francisco de Miranda (UNEFM). Santa Ana de Coro. Venezuela

\section{Elkin Baleta-Araujo; Aneleth Jasbleydi Olmedo-Plata}

- El éxito empresarial dependerá de la habilidad de los gerentes para integrarse con sus clientes y proveedores en una red compleja de relaciones en un marco de administración efectiva de la cadena de suministros, lo cual, será la clave para competir en un mundo globalizado e interconectado.

- Debe haber un gran compromiso entre el gobierno, el sector educativo, las empresas de tecnología, los gremios industriales, las compañías consultoras en logística y administración de la cadena de suministros y la industria como actor principal para coordinar adecuadamente cada eslabón si se quiere que este proceso mejore considerablemente el nivel competitivo de las empresas colombianas.

- Hay tres aspectos que han impedido no solamente en Colombia sino también en la mayor parte de los países de América Latina que las prácticas de la cadena de suministros no hayan desarrollado todo su potencial: la falta de una adecuada educación en lo que realmente, significa e implica para las empresas trabajar con estos nuevos esquemas; la incoherencia y desorden en la aplicación de metodologías más por estar a la moda que por aplicar un sentido de competencia global y la falta de un proceso evolutivo sistemático y ordenado que ha propiciado la implementación de estrategias aisladas sin ningún beneficio para las cadenas totales.

- Cuando las grandes cadenas de venta se unan con sus proveedores y logren sinergias que permitan que todos ganen, se desarrollen y crezcan, y cuando el sector agrícola se integre con el sector industrial e implementen criterios y prácticas administrativas de las cadenas de suministro para que puedan competir en cualquier escenario, como por ejemplo en el ALCA (Acuerdo de Libre Comercio de las Américas ) con menores riesgos, el sector empresarial estará recorriendo los caminos de la reactivación económica y en consecuencia buscando mejorar la calidad de vida de los colombianos. 


\section{Elkin Baleta-Araujo; Aneleth Jasbleydi Olmedo-Plata}

\section{REFERENCIAS CONSULTADAS}

Arenas, F. (2011) "Repensando su Cadena de Valor". Instituto Panamericano de Alta Dirección (IPADE). https://n9.cl/orjk

Ballesteros, D y Ballesteros, P (2014) La logística competitiva y la administración de la cadena de suministros. Scientia et Technica Año X, No 24. UTP. ISSN 01221701.

Carrasco, J. (2010): Evolución de los enfoques y conceptos de la logística "Su impacto en la dirección y la gestión de las organizaciones". Economía Industrial. No. 331.

CSCMP (2010). Supply Chain and Logistics Terms and Glossary. Council of Supply Chain Management Professional. Recuperado de: www.cscmp.org.

Eskandari, H., Sala-Diakanda, S., Furterer, S., Rabelo, L., Crumpton-Young, L y Williams, K., (2007). Enhancing the undergraduate industrial engineering curriculum: Defining desired characteristics and emerging topics. Education + Training, Vol. 49 Iss: 1, $45-55$.

Fernández J. Carlos (2008). Administración de operaciones y logística. Disponible en: https://n9.cl/s7w7

Giménez, C.(2010) "Grado de desarrollo de la gestión de la cadena de suministros y sus relaciones de colaboración en el sector de distribución español". Universidad de Barcelona, España.

Goldratt, M. (1979): Cadena Crítica. The North River Press. U.S.A.

Gutiérrez, F.; Fuquen, E.; González, H. y Hernández, A. (2010). Planificación integrada de producción y distribución para un conglomerado industria. Revista Facultad de Ingeniería Universidad de Antioquia, 53, 88-105.

Jiménez, J y Hernández, S (2012) Marco conceptual de la cadena de suministro: Un nuevo enfoque logístico. Coordinación Operativa y catedrático de la Universidad Autónoma del Estado de México.

Krugman, P.(2012) "Geografía y Comercio". Antoni Bosch. España.

Marskell, B.H.(2008) “New Performance Measure” Productivity Press, Oregon.

Martínez, L., \& El Kadi, O. (2019). Logística Integral y Calidad Total, Filosofía de Gestión Organizacional orientadas al cliente. Revista Arbitrada Interdisciplinaria Koinonía, 4(7), 202-232. http://dx.doi.org/10.35381/r.k.v4i7.201 


\section{Elkin Baleta-Araujo; Aneleth Jasbleydi Olmedo-Plata}

Nickl, M. (2005). La evolución del concepto "Logística" al de "Cadena de Suministros" y más allá. Revista Compras y Existencias, 140, 15-19.

Porter, M. E. (2010) "Ventaja Competitiva: creación y sostenimiento de un desempeño superior". Compañía Editorial Continental, 19va impresión. México.

Quiroga, J. (2009). Introducción a la logística, La logística como herramienta de competitividad. Recuperado de: https://n9.cl/0wlt

Tamayo, M. (2009). El proceso de la investigación científica. (5ta edición). México: Editorial Limusa.

Urrego-Cruz, L. (2020). Gerencia cuántica como estrategia para el desarrollo de la espiritualidad a través de los neurotalentos. CIENCIAMATRIA, 6(11), 132-149. https://doi.org/10.35381/cm.v6i11.330

Vivar-Astudillo, A., Erazo-Álvarez, J., \& Narváez-Zurita, C. (2020). La cadena de valor como herramienta generadora de ventajas competitivas para la Industria Acuícola. Revista Arbitrada Interdisciplinaria Koinonía, 5(10), 4-33. http://dx.doi.org/10.35381/r.k.v6i10.686

Young, R. y Esqueda, P. (2015). Vulnerabilidad de la cadena de suministro: consideraciones para el caso de América Latina. Academia. Revista Latinoamericana de administración, 34, 63-78. Universidad de los Andes. Bogotá, Colombia. 